The J ournal of Nonlinear S cience and Applications

http://www.tjnsa.com

\title{
S-COINCIDENCE AND S-COMMON FIXED POINT THEOREMS FOR TWO PAIRS OF SET-VALUED NONCOMPATIBLE MAPPINGS IN METRIC SPACE
}

\author{
HONG GANG LI*
}

\begin{abstract}
In this work, the new concepts, normal product of two set-valued mappings, s-weakly compatible, s-common fixed point and the $\left(E A_{s}\right)$ property for two pairs of setvalued mappings are introduced, and the s-common fixed point existence theorems for two pairs of set-valued noncompatible mappings under strict contractive condition are proved, without appeal to continuity of any map involved therein and completeness of underlying space. The results presented in this paper generalize, improve, and unify some recent results in this field.
\end{abstract}

\section{INTRODUCTION}

The problem for common fixed point is an important and interesting, have wide applications to many fields in the mathematic. For these reasons, various variational inclusions have been intensively studied in recent years. In 1994, Pant[18] initiated the study of noncompatible maps satisfying certain contractive conditions, and afterwards Aamri and El Moutawakil[3] defined a property (EA) for single valued maps on a metric space and obtained some common fixed point theorems for such maps under strict contractive conditions. The class of mappings satisfying (EA) property contains compatible as well as noncompatible maps. Kamran extended the property (EA) for a hybrid pair of single valued and set-valued maps in the [12]. Y. Liu et al. [16] obtained coincidence and common fixed point results for two pairs of hybrid maps defining common (EA) property for such pairs. On the other hand, in 1982, Sessa[20] introduced the concept of weakly commuting maps. Jungck[7]

Date: Received: 22 Sep. 2009.

* Corresponding author.

2000 Mathematics Subject Classification. 49J40, 47H06.

Key words and phrases. Normal product; s-coincidence point for two pairs of set-valued mappings; scommon Fixed Point Theorems; s-noncompatible; s-weakly compatible; $\left(E A_{s}\right)$ property. 
generalized the notion of weak commutativity by introducing compatible maps and then weakly compatible maps[8]. Jungck and Rhoades [9] further extended weak compatibility to the setting of single valued and multivalued maps. Since then, many interesting coincidence and common fixed point theorems of compatible and weakly compatible maps under various contractive conditions and assuming the continuity of at least one of the mappings, have been obtained by a number of authors. Recently, Ismat Beg and Mujahid Abbas [4 have discussed and studied the fixed point theorems for two hybrid pairs of single valued and multivalued noncompatible maps.

The aim of this paper is introduce to some new concepts, normal product of two set-valued mappings, s-weakly compatible, s-common fixed point and the $\left(E A_{s}\right)$ property for two pairs of set-valued mappings are introduced, and the s-common fixed point existence theorems for two pairs of set-valued noncompatible mappings under strict contractive condition are proved, without appeal to continuity of any map involved therein and completeness of underlying space which extend, unify and improve the earlier comparable results of a number of authors(see, [1]-[14], [16]-[18]). we refer to [1]-[23] and references contained therein.

\section{Preliminaries}

Let $(X, d)$ be a metric space. We denoted by $C B(X)$ the family of all nonempty closed bounded subsets of $X$. For $x \in X$ and $A \subseteq X, d(x, A)=\inf \{d(x, y): y \in A\}$. Let $H$ be a Hausdorff metric induced by the metric $d$ of $X$, that is,

$$
H(A, B)=\max \left\{\sup _{x \in A} d(x, B), \sup _{y \in B} d(y, A)\right\}, \quad \text { for } \quad A, B \in C B(X) .
$$

Let $2^{X}$ denote the family of all the nonempty subsets of $X, C B(X)$ denote the family of all nonempty closed bounded subsets of $X$ and $T: X \rightarrow 2^{X}$ be a set-valued mapping and $T x=T(x)$ for $x \in X$. Let us show some concepts and results.

Definition 2.1. Let $P, T: X \rightarrow 2^{X}$, then $P T x=\{P(y): y \in T(x), \forall x \in X\} \subseteq 2^{X}$ denote a product of $P$ and $T$. A product $P T x$ is said to be a normal product, if $P, T: X \rightarrow C B(X)$ then $P T x \subseteq C B(X)$ for any $x \in X$.

It is easy to see that $P T x \neq T P x$ for $x \in X$ in general.

Lemma 2.2. Let $2^{X}$ denote the family of all the nonempty subsets of $X$, and $G, P, T: X \rightarrow$ $2^{X}$ be three set-valued mappings, then for $x \in X$, the following relations hold:

(1) $P(G \bigcup T) x=P G x \bigcup P T x$;

(2) $(G \bigcup T) P x=G P x \bigcup T P x$;

(3) $P(G \bigcap T) x=P G x \bigcap P T x$;

(4) $(G \bigcap T) P x=G P x \bigcap T P x$;

(5) if $\bar{T} x=X-T x$ denote a complement of the mapping $T x$ for any $x \in X$, then $\bar{P} T x=\overline{P T x}$.

Proof.. This directly follows from the definitions of the product and the complement.

Definition 2.3. Let $P, T: X \rightarrow C B(X)$. A point $x \in X$ is said to be:

(1) fixed point of $P$ if $x \in P(x)$;

(2) $S$-coincidence point of a pair $(P, T)$ if $P x \subseteq T x$; 
(3) S-common fixed point of a pair $(P, T)$ if $\{x\} \subseteq P x \cap T x$.

$\boldsymbol{F}_{s}(P), \boldsymbol{C}_{s}(P, T)$ and $\boldsymbol{F}_{s}(P, T)$ denote set of all fixed points of $P$, set of all coincidence points of the pair $(P, T)$ and the set of all common fixed points of the pair $(P, T)$, respectively.

Definition 2.4. Let $P, X: \rightarrow C B(X)$ be two set valued mappings, and a product $P T$ be a normal product. Set valued mappings $P, T$ are said to be:

(4) S-compatible if $H\left(P y_{n}, T z_{n}\right) \rightarrow 0$ for any $y_{n} \in T x_{n}$ and any $z_{n} \in P x_{n}$ whenever $\left\{x_{n}\right\}$ is a sequence in $X$ such that $\lim _{n \rightarrow \infty} P x_{n}=\sigma \subseteq \lim _{n \rightarrow \infty} T x_{n}=A \in C B(X)$.

(5) S-noncompatible if there exists a sequence $\left\{x_{n}\right\}$ in $X$ such that $\lim _{n \rightarrow \infty} P x_{n}=\sigma \subseteq$ $\lim _{n \rightarrow \infty} T x_{n}=A \in C B(X)$, but $\lim _{n \rightarrow \infty} H\left(P y_{n}, T z_{n}\right) \neq 0$ for any $y_{n} \in T x_{n}$ and any $z_{n} \in P x_{n}$, or nonexistent.

Definition 2.5. Let $P, X: \rightarrow C B(X)$ be two set valued mappings, and a product $P T$ be a normal product. The pair $(P, T)$ is called:

(6) $S$-commuting if $T P x=P T x$ for all $x \in X$;

(7) S-weakly compatible if they commute at their coincidence points, that is, PTx $=T P x$ whenever $x \in \boldsymbol{C}_{s}(P, T)$;

(8) $(\text { IT })_{s}$-commuting at $x \in X$ if PT $\subseteq$ TP $x$.

Definition 2.6. Let $P, T: X \rightarrow C B(X)$. The set valued map $P$ is said to be $T$-weakly $S$-commuting at $x \in X$ if $P y \subseteq T y$ for any $y \in P x$.

Definition 2.7. Mappings $P, T: X \rightarrow C B(X)$ are said to satisfy property $\left(E A_{s}\right)$ if there exists a sequence $\left\{x_{n}\right\}$ in $X$, some $\sigma \subseteq X$, and $A \in C B(X)$ such that $\lim _{n \rightarrow \infty} P x_{n}=\sigma \subseteq$ $\lim _{n \rightarrow \infty} T x_{n}=A \in C B(X)$

Now we present an example of set valued mapping pair $\{P, T\}$ which satisfies $\left(E A_{s}\right)$ property and $P$ is T weakly S-commuting at some $x \subseteq \mathbf{C}_{s}(P, T)$.

Example 2.8. Let $X=[0, \infty)$ with usual metric. Define $P, T: X \rightarrow C B(X)$ by

$$
P x= \begin{cases}\{0\}, & 0 \leq x<1 \\ {[1,1+x],} & 1 \leq x<\infty\end{cases}
$$

and

$$
T x=\left\{\begin{array}{lr}
{[0, x],} & 0 \leq x<1 \\
{[1,2+x],} & 1 \leq x<\infty
\end{array}\right.
$$

It can be easily verified that the product $P T$ be a normal product, the pair $\{P, T\}$ satisfies $\left(E A_{s}\right)$ property and $P$ is $T$-weakly S-commuting at $x=0 \in \mathbf{C}_{s}(P, T)$. Moreover, $F_{s}(P, T) \neq$ $\emptyset$.

Lemma 2.9. ([5]) Let $A, B \in C B(X)$, then for any $x \in A, d(x, B) \leq H(A, B)$.

\section{S-Common Fixed Point}

The following result extends Theorem 2.1 of [4], and of course, extends Theorem 1 of [22], Theorem 3 of [11] and improves Theorem 2.3 of [4]. 
Theorem 3.1. Let $(X, d)$ be a metric space, $G, P, Q, T: X \rightarrow C B(X)$ be set valued mappings, and the products $P T$ and $G Q$ be two normal products. If the pair $\{G, Q\}$ satisfies $\left(E A_{s}\right)$ property, $G(X) \subseteq P(X) \subseteq C B(X)$ and there exist, $r \in[0,1), p \in P x$ and $G y \in C B(X)$ for all $x, y \in X, x \neq y$ such that

$$
H(T x, Q y)<\max \left\{d(p, G y), r d(p, T x), r H(G y, Q y), \frac{1}{2}[d(p, Q y)+H(G y, T x)]\right\}
$$

then the pair $\{P, T\}$ and pair $\{G, Q\}$ have $S$-coincidence points. Moreover, $P, G, T$ and $Q$ have a $S$-common fixed point if $P$ is $T$-weakly $S$-commuting at $x \in C_{s}(f, T)$ and $G$ is $Q$-weakly S-commuting at $y \in \boldsymbol{C}_{s}(G, T)$.

Proof.. Since the pair $\{G, Q\}$ satisfies property $\left(E A_{s}\right)$, there exist a sequence $\left\{x_{n}\right\}$ in $X$ and $\sigma, D \subseteq C B(X)$ such that $\lim _{n \rightarrow \infty} G x_{n}=\sigma \subseteq D=\lim _{n \rightarrow \infty} Q x_{n} \in C B(X)$. Since, $G(X) \subseteq P(X) \subseteq C B(X)$, for each $x_{n}$, there exists $y_{n} \in X$ such that $P y_{n}=G x_{n}$. Therefore, $\lim _{n \rightarrow \infty} P y_{n}=\lim _{n \rightarrow \infty} G x_{n}=\sigma \subseteq D=\lim _{n \rightarrow \infty} Q x_{n} \in C B(X)$. Since $\sigma \in P(X) \cap G(X)$, there exists $u, v \in X$ such that $\sigma=P u=G v$. We claim that $P u \subseteq T u$. If not, then there exists a element $p \in P u-T u$, and $H(P u, T u) \geq H(p, T u)>0$ for $T u \in C B(X)$. By condition (3.3), we have,

$$
\begin{aligned}
& H\left(T u, Q x_{n}\right) \leq \max \left\{d\left(p, G x_{n}\right), r d(p, T u), r H\left(G x_{n}, Q x_{n}\right), \frac{1}{2}\left[d\left(p, Q x_{n}\right)+H\left(G x_{n}, T u\right)\right]\right\} \\
& \leq \max \left\{H\left(P u, G x_{n}\right), r H(P u, T u), r H\left(G x_{n}, Q x_{n}\right), \frac{1}{2}\left[H\left(P u, Q x_{n}\right)+H\left(G x_{n}, T u\right)\right]\right\},
\end{aligned}
$$

where $r \in[0,1)$ and $p \in P u$.

Taking limit $n \rightarrow \infty$, we have

$$
\begin{aligned}
& H(T u, D) \leq \max \left\{H(P u, \sigma), r H(P u, T u), r H(\sigma, D), \frac{1}{2}[H(P u, D)+H(D, T u)]\right\} \\
& \leq \max \left\{r H(P u, T u), \frac{1}{2} H(\sigma, T u)\right\} .
\end{aligned}
$$

It further implies that

$$
H(P u, T u)=H(\sigma, T u) \leq H(D, T u) \leq \max \left\{r H(P u, T u), \frac{1}{2} H(P u, T u)\right\}
$$

and $H(P u, T u)=0$, which is a contradiction. Thus $P u \subseteq T u$.

Now we show that $\lim _{n \rightarrow \infty} T y_{n}=D$. Otherwise, there exists a positive real number $\varepsilon$, positive integer $N$, and a subsequence $\left\{T y_{n_{k}}\right\}$ of $\left\{T y_{n}\right\}$ such that $H\left(T y_{n_{k}}, D\right) \geq \varepsilon$, for $n_{k} \geq N$. From 
assumption and the Lemma 2.7, it follows that

$$
\begin{aligned}
& H\left(T y_{n_{k}}, D\right) \leq H\left(T y_{n_{k}}, Q x_{n_{k}}\right)+H\left(Q x_{n_{k}}, D\right) \\
& \leq \max \left\{d\left(p_{k}, G x_{n_{k}}\right), r d\left(p_{k}, T y_{n_{k}}\right), r H\left(G x_{n_{k}}, Q x_{n_{k}}\right),\right. \\
& \left.\frac{1}{2}\left[d\left(p_{k}, Q x_{n_{k}}\right)+H\left(G x_{n_{k}}, T y_{n_{k}}\right)\right]\right\}+H\left(Q x_{n_{k}}, D\right), \\
& \leq H\left(T y_{n_{k}}, Q x_{n_{k}}\right)+H\left(Q x_{n_{k}}, D\right) \\
& \leq \max \left\{H\left(P y_{n_{k}}, G x_{n_{k}}\right), r H\left(P y_{n_{k}}, T y_{n_{k}}\right), r H\left(G x_{n_{k}}, Q x_{n_{k}}\right)+H\left(Q x_{n_{k}}, D\right),\right. \\
& \left.\frac{1}{2}\left[H\left(P y_{n_{k}}, Q x_{n_{k}}\right)+H\left(G x_{n_{k}}, T y_{n_{k}}\right)\right]\right\}+H\left(Q x_{n_{k}}, D\right),
\end{aligned}
$$

where $p_{k} \in P y_{n_{k}}, G x_{n_{k}} \subseteq C B(X)$.

Apply limit $k \rightarrow \infty$,

$$
\lim _{n \rightarrow \infty} H\left(T y_{n_{k}}, \sigma\right) \leq \lim _{n \rightarrow \infty} H\left(T y_{n_{k}}, D\right) \leq \max \left\{r \lim _{n \rightarrow \infty} H\left(\sigma, T y_{n_{k}}\right), \frac{1}{2} \lim _{n \rightarrow \infty} H\left(\sigma, T y_{n_{k}}\right)\right\}
$$

which is a contradiction. Hence $\lim _{n \rightarrow \infty} T y_{n}=D$.

We can show that $G v \subseteq Q v$. In the face, if not, then for any $g \in G v-Q v$ and $Q v \in C B(X)$, $H(G v, Q v) \geq d(g, Q v)>0$. By condition (3.3), we have,

$$
H\left(T y_{n}, Q v\right) \leq \max \left\{H\left(P y_{n}, G v\right), r H\left(P y_{n}, T y_{n}\right), r H(G v, Q v), \frac{1}{2}\left[H\left(P y_{n}, Q v\right)+H\left(G v, T y_{n}\right)\right]\right\}
$$

where $r \in[0,1)$ and $p_{n} \in P y_{n}$.

Taking limit $n \rightarrow \infty$, we have

$$
\begin{aligned}
& H(D, Q v) \leq \max \left\{H(\sigma, G v), r H(\sigma, D), r H(G v, Q v), \frac{1}{2}[H(\sigma, Q v)+H(G v, D)]\right\} \\
& \leq \max \left\{r H(G v, Q v), \frac{1}{2} H(G v, Q v)\right\} .
\end{aligned}
$$

It further implies that

$$
H(G v, Q v)=H(\sigma, Q v) \leq H(D, Q v) \leq \max \left\{r H(G v, Q v), \frac{1}{2} H(G v, Q v)\right\},
$$

and $H(G v, Q v)=0$, which is a contradiction. Thus $G v \subseteq Q v$.

Now, we show that $\{u, v\} \subseteq P u \cap T u \cap G v \cap Q v$. $P, G, T$ and $Q$ have a S-common fixed point. By assumption, $P^{2} u \subseteq T P u$ and $G^{2} v \subseteq Q G v$ because that $P$ is $T$-weakly S-commuting at $u \in \mathbf{C}_{s}(f, T)$ and $G$ is $Q$-weakly S-commuting at $v \in \mathbf{C}_{s}(G, T)$. Also, using the Lemma 2.7, we obtain, $H(P u, G g) \leq H(T u, Q g)$ for any $g \in G v$. We claim that $u \in P u$. 
If not, then condition (3.3) implies that

$$
\begin{aligned}
& H(T u, Q p)<\max \left\{d(p, G p), r d(p, T u), r H(G p, Q p), \frac{1}{2}[d(g, Q p)+H(G p, T u)]\right\}, \\
& \leq \max \left\{H(P u, G p), r H(P u, T u), r H(G p, Q p), \frac{1}{2}[H(G p, Q p)+H(G p, T u)]\right\} \\
& \leq \max \left\{H(T u, Q p), r H(P u, T u), r H(Q p, Q p), \frac{1}{2}[H(Q p, Q p)+H(Q p, T u)]\right\} \\
& =H(T u, Q p)
\end{aligned}
$$

for $p \in P u=G v$ and any $g \in G p \subseteq Q p$, which is a contradiction and the claim follows. And we claim that $v \in G v$ as same as the way. It further implies $\{u, v\} \subseteq P u \cap T u \cap G v \cap Q v$.

Lastly, we claim that $u=v$. If not, then condition (3.3) implies that

$$
\begin{aligned}
& H(T u, Q v)<\max \left\{d(p, G v), r d(p, T u), r H(G v, Q v), \frac{1}{2}[d(p, Q v)+H(G v, T u)]\right\} \\
& \leq \max \left\{H(P u, Q v), r H(P u, T u), r H(G v, Q v), \frac{1}{2}[H(G v, Q v)+H(G v, T u)]\right\} \\
& \leq \max \left\{H(T u, Q v), r H(P u, T u), r H(G v, Q v), \frac{1}{2}[H(G v, Q v)+H(Q v, T u)]\right\} \\
& =H(T u, Q v)
\end{aligned}
$$

where $r \in[0,1), p \in P u=G v \subseteq Q v$, which is again a contradiction and the claim follows. As was stated above the $\{u\} \subseteq P u \cap G u \cap T u \cap Q u$, that is, $P, G, T$ and $Q$ have a S-common fixed point $u$. This completes the proof.

Corollary 3.2. Let $(X, d)$ be a metric space, $P, T, G, Q: X \rightarrow C B(X)$ be set-valued mappings. The pair $\{G, Q\}$ is S-noncompatible, $G(X) \subseteq P(X) \subseteq C B(X)$ and there exist, $r \in[0,1), p \in P x$ and $G y \in C B(X)$ for all $x, y \in X, x \neq y$ such that

$$
H(T x, Q y)<\max \left\{d(p, G y), r d(p, T x), r H(G y, Q y), \frac{1}{2}[d(p, Q y)+H(G y, T x)]\right\},
$$

then the pair $\{P, T\}$ and pair $\{G, Q\}$ have $S$-coincidence points. Moreover, $P, G, T$ and $Q$ have a $S$-common fixed point if $P$ is $T$-weakly $S$-commuting at $x \in C_{s}(f, T)$ and $G$ is $Q$-weakly S-commuting at $y \in \boldsymbol{C}_{s}(G, T)$.

Let $P=f$ and $G=g$ be two single-valued mappings, then corollary 3.2 extends corollary 2.2 of [4], to set valued mappings.

Remark 3.3. Let $(X, d)$ be a metric space, $P, T, G, Q: X \rightarrow C B(X)$ be set-valued mappings. The pair $\{G, Q\}$ satisfies $\left(E A_{s}\right)$ property, $G(X) \subseteq P(X) \subseteq C B(X)$, If taking $1 \geq r \geq \frac{1}{2}$ in Theorem 3.1, and for $p \in P x, G y \in C B(X)(\forall x, y \in X, x \neq y)$ such that

$$
H(T x, Q y)<\max \left\{d(p, G y), d(p, T x), H(G y, Q y), \frac{1}{2}[d(p, Q y)+H(G y, T x)]\right\},
$$

then pairs $\{P, T\}$ and $\{G, Q\}$ have $S$-coincidence points. Moreover, $P, G, T$ and $Q$ have a $S$-common fixed point if $P$ is $T$-weakly $S$-commuting at $x \in \boldsymbol{C}_{s}(f, T)$ and $G$ is $Q$-weakly $S$-commuting at $y \in \boldsymbol{C}_{s}(G, T)$. 
Let $\varphi:(0,+\infty) \rightarrow(o,+\infty)$ be a continuous and nondecreasing function such that $0<$ $\varphi(t)<t$ for each $t \in(0,+\infty)$. The following corollary improves Theorem 2.5 of [12], Theorem 2.10 of [16], and Theorem 2.1 of [4].

Corollary 3.4. Let $(X, d)$ be a metric space, $G, P, Q, T: X \rightarrow C B(X)$ be set valued mappings. If the pair $\{G, Q\}$ satisfies $\left(E A_{s}\right)$ property, $G(X) \subseteq P(X) \subseteq C B(X)$ and there exist, $r \in[0,1), p \in P x$ and $G y \in C B(X)$ for all $x, y \in X, x \neq y$ such that

$$
H(T x, Q y)<\varphi\left(\max \left\{d(p, G y), r d(p, T x), r H(G y, Q y), \frac{1}{2}[d(p, Q y)+H(G y, T x)]\right\}\right),
$$

then the pair $\{P, T\}$ and pair $\{G, Q\}$ have $S$-coincidence points. Moreover, $P, G, T$ and $Q$ have a $S$-common fixed point if $P$ is $T$-weakly $S$-commuting at $x \in C_{s}(f, T)$ and $G$ is $Q$-weakly $S$-commuting at $y \in \boldsymbol{C}_{s}(G, T)$.

Proof. The proof directly follows from the definition of $\varphi:(0,+\infty) \rightarrow(o,+\infty)$ and the method proved Theorem 3.2, and so it is omitted.

Acknowledgments The authors acknowledgment support of the Educational Science Foundation of Chongqing, Chongqing(KJ091315).

\section{REFERENCES}

[1] A. Azam, M. Arshad and I. Beg, Common fixed point theorems in cone metric spaces, The Journal of Nonlinear Science and its Applications, Vol. 2 No. 4, (2009) pp. 204-213.

[2] A. Azam and I. Beg, Coincidence point ofcompatible multivalued mappings, Demonstratio Mathematica, 29 (1996), 17-22.

[3] M. Aamri and D. El Moutawakil, Some new common fixed point theorems under strict contractive conditions, J. Math. Anal. Appl., 270 (2002), 181- 188.

[4] I. Beg and M. Abbas, Coincidence and fixed point theorems for two hybrid pairs of noncompatible maps, Nonlinear Analysis Series A, in preen

[5] L. S. Dube, A theorem on common fixed points of multivalued mappings, Annal. Soc. Sci. Bruxells, 84 (1975), 463-468.

[6] O. Hadzic, Common fixed point theorem for single valued and multivalued mappings, Rev. of Research for Sci. Math. Series., 18 (1988), 145-151.

[7] G. Jungck, Common fixed points for commuting and compatible maps on compacta, Proc. Amer. Soc., 103 (1988), 977-983.

[8] G. Jungck, Common fixed points for noncontinuous nonself maps on a nonmetric space, Far East J. Math. Sci., 4(2) (1996), 199-212.

[9] G. Jungck and B. E. Rhoades, Fixed points for set valued functions without continuity, Indian J. Pure Appl. Math., 29 (1998), 227-238.

[10] G. Jungck, Commuting mappings and fixed points, Amer. Math. Monthly, 83 (1976), 261-263.

[11] H. Kaneko and S. Sessa, Fixed point theorems for compatible multivalued and single valued mappings, Int. J. Math. Math. Sci., 12 (1989), 257-267.

[12] T. Kamran, Coincidence and fixed points for hybrid strict contractions, J. Math. Anal. Appl., 299 (2004), 235-241.

[13] H. G. Li, Perturbed Ishikawa Iterative Algorithm and Stability for Nonlinear Mixed Quasi-Variational Inclusions Involving $(A, \eta)$-accretive Mappings, Advances in Nonlinear Variational Inequalities, 11(1) (2008), 41-50.

[14] H. G. Li, Approximate algorithm of solutions for general nonlinear fuzzy mulitvalued quasi-varaiational inclusions with $(G, \eta)$-monotone mappings, Nonlinear Functional Analysis and Applications, 13(2) (2008), pp. 277-289. 
[15] Y. Li, F. Gu, Common fixed piont theorem of altman integral type mappings, The Journal of Nonlinear Science and its Applications, Vol. 2 No. 4, (2009) pp. 214-218.

[16] Y. Liu, J. Wu and Z. Li, Common fixed points of single valued and multivalued maps, Int. J. Math. Math. Sci., 19 (2005), 3045-3055.

[17] S. B. Nadler, Jr., Multivalued contraction mappings, Pacific J. Math., 30 (1969), 475-488.

[18] R. P. Pant, Common fixed point ofnoncomm uting mappings, J. Math. Anal. Appl., 188(1994), $436-440$.

[19] B. E. Rhoades, On multivalued f-nonexpansive maps, Fixed Point Theory Appl., 2 (2001), 89-92.

[20] S. Sessa, On a weak commutativity condition ofmappings in fixed point consideration, Publ. Inst. Math. Soc., 32(1982), 149-153.

[21] N. Shahzad, Invariant approximation and R- subweakly commuting maps, J. Math. Anal. Appl., $257(2001), 39-45$.

[22] S. L. Singh and Ashish Kumar, Common fixed point theorems for contractive maps, Mat. Bech., 58(2006), 85-90.

[23] S. S. Zhang, On problem of nearst common fixed point of nonexpansive mapping, Applied Mathematics and Mechanics 27(7) (2006), 775-781.

* Institute of Applied Mathematics Research Chongqing University of Posts and TeleComMunications Chongqing 400065, China

E-mail address: lihg12@126.com 